\title{
Language Differences Between Children with Down Syndrome, Familial Mental Impairment and Typically Developing Peers
}

\author{
V. Zarokanellou', PhD, A. Kotsopoulos ${ }^{2}, \mathrm{PhD}, \mathrm{M}$. Vlassopoulos ${ }^{3}, \mathrm{PhD}, \mathrm{K}$. Papanikolaou ${ }^{3}$,MD \\ ${ }^{1}$ Lecturer, Deparment of Speech-Therapy, University of Patras \\ ${ }^{2}$ Associate Professor, Deparment of Speech-Therapy, University of Patras \\ ${ }^{3}$ Associate Professor, Medical School of Athens, National and Kapodistrian University of Athens
}

\section{Introduction}

The last years it has been made a significant change in the study of language with the development of a theory which claims that the different language systems (semantics, pragmatics, phonology, morphology and syntax) are developing with different rates at special mental retarded populations, even if the mental retarded individuals show the same cognitive functioning level (IQ), proving asynchronies in their language development (Tager-Flusberg \& Sullivan, 1998). The purpose of this study was to compare the language profiles between children with mental impairment (MI) and typically developing (TD) peers.

\section{Method}

Participants: The participants were: a) 9 children with Down syndrome (DS) b) 9 children with Familial Mental Impairment (FMI) and c) two control groups (18 TD children). All participants were monolingual Greek-speaking children aged 7-10 years old. The participants with $\mathrm{Ml}$ could form sentences with at least 2-3 words. The two control groups were typically developing according to their teacher and their educational record.

Assesment: Every participant in the research underwent the same identical assessment, which included: 1) the Greek version of Raven's Progressive Matrices $A, B, A B$ (Sideridis et al., 2015), 2) the comprehension scale of the test of language comprehension and expession and selected tasks of the expression scale of the same test (Vogindroukas, 2009), 3) the Greek translation and adaptation of the Expressive One Word Picture Vocabulary Test-Revised (Kotsopoulos, 2003), 4-5) the vocabulary scale and the acoustic discrimination scale of the Athina Test (Paraskevopoulos et al., 2011), 6) the screening form of the Phonological and Phonetic Developmental Scale of the Panhellenic Organization of Speech-pathologists (Levanti et al., 1995), 7) the non word repetition scale (Porpodas, 1995), 8 ) the Greek version of the Action Picture Test (Vogindroukas et al., 2011), 9-10) the silent Phonological Representation Accuracy Judgment Task via software and the Learning Nonword Task via software (Kotsopoulos et al., 2012).

Statistical analysis:For the comparison between the different groups: a) FMI-TD, b) DS-TD, c) FMI-DS regression statistical analysis was used to assimilate the comparison groups for IQ. The groups wese similar for age and gender.

\section{Bibliography}

KOTSOPOULOS, A., 2003, Translation and adaptation in

LEVANTI, E. et al.., 1995, Test of Phonological and Phonetic Development in Greek. PANHELLIC ORGANIZATION OF LOGOPEDISTS. Athens.

PARASKEVOPOULOS, J. et al., 1999, ATHINA test, assessment of learning difficulties. GREEK LETTERS, Athens.

PORPODAS, K., 1995, Analysis and exploration of phonological's awareness role in acquisition of reading and writting in Greek students. General secreteriat of science and technology. Athens.

SIDERIDIS, G. et al., 2015, Raven's educational CPM/CVS. MOTIVO, Athens TAGER-FLUSBERG, $\mathrm{H}$. and SULLIVAN K. Early language development in children with mental retardation. In: Burack AJ, Hodapp MR, Zigler E. eds Handbook of mental retardation and development. New York, USA: Cambridge University Press, 1998; 8: 208-39.

\begin{tabular}{|c|c|c|c|c|c|c|}
\hline \multicolumn{7}{|c|}{ Results } \\
\hline \multirow{3}{*}{$\begin{array}{c}+ \text { Student's t-test, }{ }^{*} \text { Regression } \\
\text { statistical analysis taking into } \\
\text { consideration the different IQ levels, } \\
\text { RS=Raw Score } \\
\text { Language Tests }\end{array}$} & \multicolumn{4}{|c|}{ Groups } & \multirow[b]{3}{*}{$\mathbf{P +}$} & \multirow[b]{3}{*}{$\mathbf{P}^{*}$} \\
\hline & \multicolumn{2}{|c|}{$\begin{array}{l}\text { FMI Group } \\
\quad(\mathrm{N}=9)\end{array}$} & \multicolumn{2}{|c|}{$\begin{array}{c}\text { Control Group } \\
(\mathrm{N}=9)\end{array}$} & & \\
\hline & Mean & SD & Mean & SD & & \\
\hline Language Comprehension Scale (RS) & 145.0 & 9.4 & 162.4 & 3.1 & $<0.001$ & 0.00 \\
\hline Language Expression Scale (RS) & 25.0 & 10.3 & 42.1 & 2.8 & $<0.001$ & 0,012 \\
\hline Action Picture Test (Total RS) & 58.4 & 21.8 & 89.9 & 8.1 & 0.001 & 0.064 \\
\hline $\begin{array}{l}\text { Expression One Word Vocabulary } \\
\text { Test-Revised (RS) }\end{array}$ & 33.2 & 16.2 & 66.1 & 8.7 & $<0.001$ & 0.004 \\
\hline Athina Test (Definicions Scale) (RS) & 9.7 & 8.4 & 27.6 & 6.6 & $<0.001$ & 0.006 \\
\hline $\begin{array}{l}\text { Athina Test-Discrimination Phonemes } \\
\text { Scale (RS) }\end{array}$ & $1 ., 6$ & 7.6 & 28.0 & 1.0 & $<0.001$ & 0.001 \\
\hline Number of Phonological Errors & 0.0 & 0.0 & 30.8 & 25.2 & 0.002 & 0.04 \\
\hline $\begin{array}{l}\text { Phonological Representation } \\
\text { Accuracy Judgment Task (RS \%) }\end{array}$ & 94.8 & 4.7 & 61.1 & 30.6 & 0.005 & 0.04 \\
\hline Non-word Learning Task (RS \%) & 95.4 & 3.9 & 63.0 & 25.4 & 0.002 & 0.047 \\
\hline Non-word Repetition Scale (RS \%) & 83.8 & 20.8 & 30.6 & 32.1 & 0.001 & 0.030 \\
\hline \multirow{2}{*}{$\begin{array}{l}+\quad \text { Student's t-test, }{ }^{*} \text { Regression } \\
\text { statistical analysis taking into } \\
\text { consideration the different IQ levels, } \\
\text { RS=Raw Score }\end{array}$} & \multicolumn{4}{|c|}{ Groups } & & \\
\hline & \multicolumn{2}{|c|}{$\begin{array}{c}\text { Down Group } \\
(\mathrm{N}=9)\end{array}$} & \multicolumn{2}{|c|}{$\begin{array}{c}\text { Control } \\
\text { Group } \\
(\mathrm{N}=9)\end{array}$} & & \\
\hline Language Tests & Mean & SD & Me & SD & $\mathbf{P +}$ & $\mathbf{P}^{*}$ \\
\hline Language Comprehension Scale (RS) & 124.9 & 14.1 & 162.4 & 2.1 & $<0.001$ & $<0.001$ \\
\hline Language Expression Scale (RS) & 9.0 & 3.0 & 42.6 & 2.1 & $<0.001$ & $<0.001$ \\
\hline Action Picture Test (Total RS) & 32.9 & 18.1 & 95.4 & 8.8 & $<0.001$ & $<0.001$ \\
\hline $\begin{array}{l}\text { Expression One Word Vocabulary Test- } \\
\text { Revised (RS) }\end{array}$ & 20.9 & 11.6 & 70.7 & 4.2 & $<0.001$ & $<0.001$ \\
\hline Athina Test (Definicions Scale) (RS) & 2.1 & 1.8 & 31.3 & 3.6 & $<0.001$ & $<0.001$ \\
\hline $\begin{array}{l}\text { Athina Test-Discrimination Phonemes } \\
\text { Scale (RS) }\end{array}$ & 6.2 & 9.7 & 27.2 & 1.9 & $<0.001$ & $<0.001$ \\
\hline Vumber of Phonological Errors & 64.6 & 2.6 & 0.1 & 0.3 & $<0.001$ & $<0.001$ \\
\hline $\begin{array}{l}\text { Phonological Representation Accuracy } \\
\text { Judgment Task (RS \%) }\end{array}$ & 27.0 & 19.8 & 93.0 & 4.2 & $<0.001$ & $<0.001$ \\
\hline Von-word Learning Task (RS \%) & 23.6 & 30.1 & 88.9 & 7.8 & $<0.001$ & $<0.001$ \\
\hline Non-word Repetition Scale (RS \%) & 9.7 & 14.6 & 87.5 & 12.5 & $<0.001$ & $<0.001$ \\
\hline
\end{tabular}

\begin{tabular}{|c|c|c|c|c|c|c|}
\hline \multirow{3}{*}{$\begin{array}{l}\text { *Standard error, when it was taken into } \\
\text { consideration the different IQ levels, } \\
\text { RS=Raw Score } \\
\text { Language Tests }\end{array}$} & \multicolumn{5}{|c|}{ Groups } & \multirow[b]{3}{*}{$\mathbf{P}$} \\
\hline & \multicolumn{2}{|c|}{$\begin{array}{l}\text { FMI Group } \\
\qquad(\mathrm{N}=9)\end{array}$} & \multicolumn{2}{|c|}{$\begin{array}{l}\text { Down } \\
\text { Group } \\
(\mathrm{N}=9)\end{array}$} & \multirow[b]{2}{*}{$\beta^{*}(\mathrm{SE})$} & \\
\hline & Mean & SD & Mean & SD & & \\
\hline Language Comprehension Scale (RS) & 145.0 & 9.4 & 124.9 & 14.1 & $-13.95(6.56)$ & 0.050 \\
\hline Language Expression Scale (RS) & 25.0 & 10.3 & 9.0 & 3.0 & $-11.49(4.04)$ & 0.012 \\
\hline Action Picture Test (Total RS) & 58.4 & 21.8 & 32.9 & 18.1 & $-6.34(8.22)$ & 0.453 \\
\hline $\begin{array}{l}\text { Expression One Word Vocabulary Test- } \\
\text { Revised (RS) }\end{array}$ & 33.2 & 16.2 & 20.9 & 11.6 & $-2.55(7.14)$ & 0.727 \\
\hline Athina Test (Definicions Scale) (RS) & 9.7 & 8.4 & 2.1 & 1.8 & $-7.56(2.86)$ & 0.018 \\
\hline $\begin{array}{l}\text { Athina Test-Discrimination Phonemes } \\
\text { Scale (RS) }\end{array}$ & $1 ., 6$ & 7.6 & 6.2 & 9.7 & $-5.87(5.18)$ & 0.274 \\
\hline Number of Phonological Errors & 0.0 & 0.0 & 64.6 & 2.6 & $13.20(11.95)$ & 0.287 \\
\hline $\begin{array}{l}\text { Phonological Representation Accuracy } \\
\text { Judgment Task (RS \%) }\end{array}$ & 94.8 & 4.7 & 27.0 & 19.8 & $-29.80(15.23)$ & 0.069 \\
\hline Non-word Learning Task (RS \%) & 95.4 & 3.9 & 23.6 & 30.1 & $-18.48(13.65)$ & 0.196 \\
\hline Non-word Repetition Scale (RS \%) & 83.8 & 20.8 & 9.7 & 14.6 & $-6.37(13.32)$ & 0.639 \\
\hline
\end{tabular}

\section{Conclusions-Take away message}

The results show that the different language systems are developing with different rates at special mental retarded populations, even if the individuals with MI show the same cognitive functioning level. The current research strongly supports the developmental model for FMI and a close phenotype-genotype relationship in different MI syndromes.

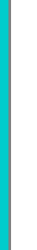

t
Copyright $\odot 2019$ Zarokanellou V. et al., vas_zaro@med.uoa.gr 